\title{
Sistema Computacional para Ajuste de Funções Densidade de Probabilidade
}

\author{
Daniel Henrique Breda Binoti ${ }^{1}$, Helio Garcia Leite ${ }^{1}$, Mayra Luiza Marques da Silva ${ }^{2}$ \\ ${ }^{1}$ Universidade Federal de Viçosa - UFV, Viçosa/MG, Brasil \\ ${ }^{2}$ Departamento de Ciências Florestais e da Madeira - DCFM, Centro de Ciências Agrárias - CCA, \\ Universidade Federal do Espírito Santo - UFES, Jerônimo Monteiro/ES, Brasil
}

\begin{abstract}
RESUMO
Este trabalho teve por objetivo iniciar, implementar e validar um projeto de construção de um sistema computadorizado para ajuste de funções densidade de probabilidade. O FitFD foi desenvolvido utilizando-se a linguagem de programação Java. Como ambiente de desenvolvimento foram utilizadas a IDE (Integrated Development Environment) Netbeans 7.1 e a JDK 7.3 (Java Development Kit). Os testes do sistema foram realizados em ambiente Windows. Foram implementadas no sistema as seguintes funções densidade de probabilidade: Weibull (2P, 3P, 2P com dap mínimo como locação, 3P truncada), hiperbólica (2P, 3P, 2P com dap mínimo como locação, 3P truncada), log-logística (2P, 3P, 2P com dap mínimo como locação), logística generalizada, Fatigue life (2P e 3P) e Frechet (2P e 3P). O sistema desenvolvido auxilia os usuários na definição e escolha da fdp que melhor atenda suas necessidades, contudo melhorias são necessárias. O projeto iniciado mostrou-se eficiente para ajustes de funções de densidade probabilidade.
\end{abstract}

Palavras-chave: FitFD, modelos de distribuição diamétrica, multiprodutos.

\section{Computer System for Adjusting the Probability Density Functions}

\begin{abstract}
This study aimed to initiate, implement and validate a project to build a computerized system to adjust probability density functions. The FitFD was developed using the Java programming language. IDE (Integrated Development Environment) NetBeans 7.1 and JDK 7.3 (Java Development Kit) were used as the development environment. The system was tested in Windows. The following probability density functions were implemented in the system: Weibull (2P, 3P, $2 \mathrm{P}$ with minimum dbh, truncated $3 \mathrm{P})$, hyperbolic (2P, 3P, $2 \mathrm{P}$ with minimum dbh, truncated 3P), Log- logistic (2P, 3P, 2P with minimum dbh), Logistics Generalized, Fatigue Life (2P and 3P) and Frechet $(2 \mathrm{P}$ and $3 \mathrm{P})$. The system developed assists users in defining and choosing fdp that best meet their needs, however improvements are needed. The project proved efficient for adjustments of probability density functions.
\end{abstract}

Keywords: FitFD, models of diameter distribution, multiproduct. 


\section{INTRODUÇÃO}

O conhecimento da distribuição diamétrica de povoamentos florestais é essencial para se inferir sobre a dinâmica de crescimento e relações entre as árvores. Isso auxilia a tomada de decisão sobre intervenções a serem realizadas no povoamento, como desbaste, desrama e colheita (Campos \& Leite, 2009), bem como permite uma análise econômica mais precisa, visto que quase a totalidade dos coeficientes operacionais das operações de colheita e transporte são influenciados pelas dimensões das árvores (Clutter et al., 1983).

A descrição das estimativas de frequência por classe diamétrica é feita pelo emprego de funções densidade de probabilidade $(f d p)$, como: Weibull, normal, log-normal, gama, Johnson's SB, beta, log-logística, Cauchy, Frechet, Erlang, Rayleigh, e hiperbólica (Binoti, 2008; Leite et al., 2010; Binoti et al., 2012). A escolha de uma dessas $f d p$ é determinante para a qualidade das estimativas da distribuição diamétrica futura (Binoti, 2008).

A recuperação da distribuição de diâmetros futura se dá pela estimação dos parâmetros da $f d p$ em função de características do povoamento como idade, densidade, índice de local, diâmetro mínimo e diâmetro máximo, dentre outras. Um das etapas mais trabalhosas para a construção de um modelo de distribuição de diâmetros consiste no ajuste das $f d p$ a cada parcela e medição. Como ferramentas gratuitas que permitem o ajuste de diferentes $f d p$ bem como a análise gráfica dos ajustes são escassas na área florestal, idealizou-se esse trabalho, o qual.teve por objetivo iniciar, desenvolver, implementar e validar um projeto de construção de um sistema computadorizado para ajuste de funções de densidade probabilidade.

\section{MATERIAL E MÉTODOS}

\subsection{Desenvolvimento do projeto}

O FitFD foi desenvolvido utilizando-se a linguagem de programação Java. Como ambiente de desenvolvimento foram utilizadas a IDE (Integrated Development Environment) Netbeans 7.1 e a JDK 7.3 (Java Development Kit). Os testes do sistema foram realizados em ambiente Windows. O sistema utiliza a biblioteca Michael Thomas Flanagan's Java Scientific Library para a obtenção dos parâmetros.

\subsection{Funções de densidade probabilidade}

Para o sistema desenvolvido optou-se pela implementação das funções de densidade probabilidade descritas abaixo.

\subsubsection{Função Weibull}

A $f d p$ da função Weibull de três parâmetros (3P) (Equação 1) pode ser descrita como:

$f(x)=\left\{\frac{\gamma}{\beta}\left(\frac{x-\alpha}{\beta}\right)^{(\gamma-1)}\left(\exp \left(-\left(\frac{x-\alpha}{\beta}\right)^{\gamma}\right)\right)\right.$

em que $\alpha$ é o parâmetro de locação, $\beta$, o parâmetro de escala $(\beta>0), \gamma, o$ parâmetro de forma $(\gamma>0)$ e $x$ é o centro de classe de diâmetro $(x>0)$. A supressão do parâmetro de locação da função caracteriza a função Weibull de dois parâmetros (2P) (Equação 2):

$f(x)=\left\{\frac{\gamma}{\beta}\left(\frac{x}{\beta}\right)^{(\gamma-1)}\left(\exp \left(-\left(\frac{x}{\beta}\right)^{\gamma}\right)\right)\right.$

O parâmetro de locação pode ser substituído pelo diâmetro mínimo do povoamento $(\alpha=d \mathrm{~min})$ (Equação 3), ajustada da seguinte forma:

$f(x)=\left\{\frac{\gamma}{\beta}\left(\frac{x-d \min }{\beta}\right)^{(\gamma-1)}\left(\exp \left(-\left(\frac{x-d \min }{\beta}\right)^{\gamma}\right)\right)\right.$

A função Weibull pode ser truncada à direita em função do diâmetro máximo do povoamento (Equação 4):

$f(x)=\left\{\frac{\frac{\gamma}{\beta}\left(\frac{x-\alpha}{\beta}\right)^{(\gamma-1)}\left(\exp \left(-\left(\frac{x-\alpha}{\beta}\right)^{\gamma}\right)\right)}{\exp \left(-\left(\frac{T-\alpha}{\beta}\right)^{\gamma}\right)}\right.$

em que $T$ é o diâmetro máximo da parcela.

\subsubsection{Função hiperbólica}

A $f d p$ da função hiperbólica de três parâmetros (3P) (Equação 5) pode ser descrita:

$f(x)=\left\{\frac{\gamma}{\beta}\left(\frac{x-\alpha}{\beta}\right)^{(\gamma-1)}\left(1-\tanh \left(\left(\frac{x-\alpha}{\beta}\right)^{\gamma}\right)^{2}\right)\right.$ 
em que, $\alpha$ é o parâmetro de locação, $\beta$, o parâmetro de escala $(\beta>0), \gamma$, o parâmetro de forma $(\gamma>0)$, e $x$, o centro de classe de diâmetro $(x>0)$. A supressão do parâmetro de locação da função caracteriza a função hiperbólica de dois parâmetros (2P) (Equação 6):

$$
f(x)=\left\{\frac{\gamma}{\beta}\left(\frac{x}{\beta}\right)^{(\gamma-1)}\left(1-\tanh \left(\left(\frac{x}{\beta}\right)^{\gamma}\right)^{2}\right)\right.
$$

O parâmetro de locação pode ser substituído pelo diâmetro mínimo do povoamento $(\alpha=d \mathrm{~min})$ (Equação 7), sendo ajustada da seguinte forma:

$$
f(x)=\left\{\frac{\gamma}{\beta}\left(\frac{x-d \min }{\beta}\right)^{(\gamma-1)}\left(1-\tanh \left(\left(\frac{x-d \min }{\beta}\right)^{\gamma}\right)^{2}\right)\right.
$$

A função hiperbólica pode ser truncada a direita em função do diâmetro máximo do povoamento (Equação 8) e ser descrita da seguinte forma:

$$
f(x)=\left\{\frac{\frac{\gamma}{\beta}\left(\frac{x-\alpha}{\beta}\right)^{(\gamma-1)}\left(1-\tanh \left(\left(\frac{x-\alpha}{\beta}\right)^{\gamma}\right)^{2}\right)}{\tanh \left(\left(\frac{T-\alpha}{\beta}\right)^{\gamma}\right)}\right.
$$

em que $T$ é o diâmetro máximo da parcela.

\subsubsection{Função log-logística}

A função densidade de probabilidade log-logística em sua forma completa (3P) (Equação 9) pode ser escrita da seguinte forma:

$$
f(x)=\left\{\frac{\gamma}{\beta}\left(\frac{x-\alpha}{\beta}\right)^{(\gamma-1)}\left(1+\left(\frac{x-\alpha}{\beta}\right)^{\gamma}\right)^{-2}\right.
$$

em que $\alpha$ é o parâmetro de forma $(\alpha>0), \beta$, o parâmetro de escala $(\beta>0)$, e $\gamma$, o parâmetro de locação, sendo que a supressão do parâmetro de locação resulta na função log-logística de dois parâmetros (2P) (Equação 10), que pode ser expressa da seguinte forma:

$$
f(x)=\left\{\frac{\gamma}{\beta}\left(\frac{x}{\beta}\right)^{(\gamma-1)}\left(1+\left(\frac{x}{\beta}\right)^{\gamma}\right)^{-2}\right.
$$

O parâmetro de locação pode também ser substituído pelo diâmetro mínimo da parcela (dapmin), resultando na função $\log$-logística com $\gamma=$ dapmin (Equação 11), como expresso a seguir:

$$
f(x)=\left\{\frac{\gamma}{\beta}\left(\frac{x-\text { dapmin }}{\beta}\right)^{(\gamma-1)}\left(1+\left(\frac{x-\text { dapmin }}{\beta}\right)^{\gamma}\right)^{-2}\right.
$$

\subsubsection{Função logística generalizada}

A função logística generalizada (Nadarajah \& Kotz, 2005) (Equação 12) pode ser descrita da seguinte forma:

$$
f(x)=\left\{\frac{\left(1+\alpha\left(\frac{x-\gamma}{\beta}\right)\right)^{-1-\frac{1}{\alpha}}}{\beta\left(1+\left(1+\alpha\left(\frac{x-\gamma}{\beta}\right)\right)^{-\frac{1}{\alpha}}\right)^{2}}\right.
$$

em que $\beta$ é o parâmetro de escala $(\beta>0)$, $\alpha$, o parâmetro de forma $(\alpha>0)$ e $\gamma$, o parâmetro de locação $(\gamma>0)$.

\subsubsection{Função Fatigue life}

A função Birnbaum-Saunders, também conhecida como Fatigue life, foi proposta por Birnbaum \& Saunders (1969a, b) para a descrição da fadiga causada por ações realizadas ciclicamente. A função Birnbaum-Saunders pode ser ajustada pelo método da máxima verossimilhança (Birnbaum \& Saunders, 1969b), por métodos bayesianos (Achar, 1993) ou pelo método de momentos (Ng et al., 2003; Wang et al., 2006).

A função densidade de probabilidade Birnbaum-Saunders, em sua forma completa (3P) (Equação 13), pode ser escrita da seguinte forma:

$$
f(x)=\left\{\frac{\sqrt{(x-\gamma) / \beta}+\sqrt{\beta /(x-\gamma)}}{2 \alpha(x-\gamma)} \phi\left(\frac{1}{\alpha}\left(\sqrt{\frac{x-\gamma}{\beta}}-\sqrt{\frac{\beta}{x-\gamma}}\right)\right)\right.
$$

em que $\alpha$ é o parâmetro de forma $(\alpha>0), \beta$, o parâmetro de escala $(\beta>0)$, $\gamma$, o parâmetro de locação e $\phi$, a função normal padrão (Equação 14), que pode ser escrita:

$\phi=\frac{e^{-x^{2} / 2}}{\sqrt{2 \pi}}$

sendo que a supressão do parâmetro de locação resulta na função Birnbaum-Saunders de 2P (Equação 15), expressa da seguinte forma:

$$
f(x)=\left\{\frac{\sqrt{x / \beta}+\sqrt{\beta / x}}{2 \alpha x} \phi\left(\frac{1}{\alpha}\left(\sqrt{\frac{x}{\beta}}-\sqrt{\frac{\beta}{x}}\right)\right)\right.
$$




\subsubsection{Função Frechet}

A função densidade de probabilidade Frechet, em sua forma completa (3P) (Equação 16), pode ser escrita da seguinte forma:

$$
f(x)=\left\{\frac{\alpha}{\beta}\left(\frac{\beta}{x-\gamma}\right)^{(\alpha+1)}\left(\exp \left(-\left(\frac{\beta}{x-\gamma}\right)^{\alpha}\right)\right)\right.
$$

em que $\alpha$ é o parâmetro de forma $(\alpha>0), \beta$, o parâmetro de escala $(\beta>0)$ e $\gamma$, o parâmetro de locação, sendo que a supressão do parâmetro de locação resulta na função Frechet de 2P (Equação 17), expressa da seguinte forma:

$$
f(x)=\left\{\frac{\alpha}{\beta}\left(\frac{\beta}{x}\right)^{(\alpha+1)}\left(\exp \left(-\left(\frac{\beta}{x}\right)^{\alpha}\right)\right)\right.
$$

\section{RESULTADOS E DISCUSSÃO}

O sistema computacional denominado FitFD - Sistema para Ajuste de Funções de Densidade Probabilidade foi desenvolvido visando ser o mais amigável possível para o usuário, admitindo certa flexibilidade na escolha das $f d p$ cadastradas e amplitude de classes, bem como oferecendo uma visualização dinâmica dos resultados. A principal característica do sistema é poder ser utilizado livremente por gestores florestais, acadêmicos e extensionistas no ajuste de funções de densidade probabilidade e construção de modelos de distribuição de diâmetros.

A linguagem Java foi escolhida por ser uma linguagem simples, orientada a objetos, e por ser robusta, segura, neutra em relação à arquitetura, portável, facilmente interpretável, de alto desempenho, de múltiplos threads e dinâmica, dentre outras características (Horstmann \& Cornell, 2010). Dentre as principais características destaca-se o fato de que ser simples permite a continuidade do projeto por outros estudantes de graduação, pós-graduação, professores ou profissionais da área. $\mathrm{O}$ fato de ser orientada a objetos permite que futuras alterações possam ser facilmente implementadas e adicionadas ao projeto, sem inferência nas funções já disponíveis.

A estrutura do sistema é relativamente simples, baseia-se na importação das informações de diâmetro observadas em campo, processamento e ajuste das $f d p$, análise gráfica e exportação dos parâmetros das $f d p$, bem como das estimativas por classe de diâmetro.
As principais características e interfaces do sistema são apresentadas a seguir. A tela inicial do FitFD é apresentada na Figura 1.

As informações básicas relacionadas às classes de diâmetro são inseridas na aba Dados (Figura 2). As informações necessárias para o ajuste das $f d p$ são fornecidas em função de uma chave primária, que pode ser em função da parcela, do talhão ou de qualquer outra unidade administrativa.

Após a importação dos dados deve-se escolher sobre quais funções deseja-se ajustar (Figura 3). As funções disponíveis são Weibull 2 Parâmetros, Weibull 3 Parâmetros, Weibull 2 Parâmetros-locação = DapMin, Weibull 3 Parâmetros Truncada à direita, Hiperbólica 2 Parâmetros, Hiperbólica 3 Parâmetros, Hiperbólica 2 Parâmetros-locação = DapMin, Hiperbólica 3 Parâmetros Truncada, Log-Logística 2 Parâmetros, Log-Logística 3 Parâmetros, Log-Logística 2 Parâmetros-locação = DapMin, Logística Generalizada, Fatigue Life 2 Parâmetros, Fatigue Life 3 Parâmetros, Frechet 2 Parâmetros, Frechet 3 Parâmetros. O sistema permite o agrupamento dos dados em classes definidas pelo usuário.

Após o ajuste das funções pode-se verificar os valores estimados para classe de diâmetro na aba Análise (Figura 4). A análise gráfica entre valores observados e estimados pode ser realizada para todas as funções e parcelas (Figura 5) - esse fato permite a escolha e análise detalhadas das $f d p$ disponíveis.

$\mathrm{Na}$ aba Resultados pode-se observar os parâmetros de cada função ajustada pelo sistema (Figura 6).

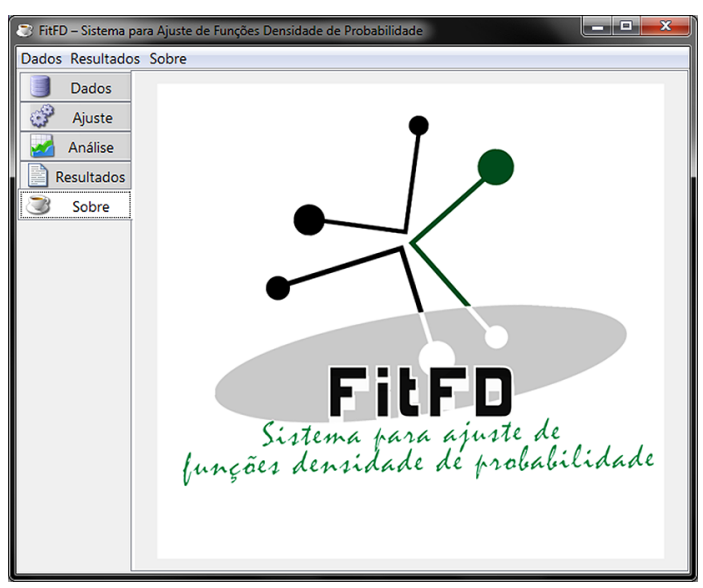

Figura 1. Tela inicial do FitFD.

Figure 1. Initial screen of FitFD. 


\begin{tabular}{|c|c|c|c|}
\hline \multicolumn{3}{|c|}{ (3) FitFD - Sistema para Ajuste de Funções Densidade de Probabilidade } & $=\quad-\quad x$ \\
\hline \multicolumn{4}{|c|}{ Dados Resultados Sobre } \\
\hline$\exists$ Dados & Chave & Dap & \\
\hline 9 & m 1iAb1trat111AP1 & 10.4 & A \\
\hline Ajuste & m1iAb1trat111AP1 & 8.4 & $\exists$ \\
\hline \multirow{4}{*}{\begin{tabular}{|l|l|} 
Análise \\
鷕 Resultados \\
\end{tabular}} & m 1iab1trat111AP1 & 6.9 & \\
\hline & m 1iAb1trat111AP1 & 9.1 & \\
\hline & m 1iAb1trat111AP1 & 8.3 & \\
\hline & m1iAb1trat111AP1 & 6.7 & \\
\hline \multirow{22}{*}{ (3) Sobre } & m1iAb1trat111AP1 & 10.3 & \\
\hline & m1iAb1trat111AP1 & 9.1 & \\
\hline & m1iAb1trat111AP1 & 7.3 & \\
\hline & m 1iAb1trat111AP1 & 9.8 & \\
\hline & m 1iAb1trat111AP1 & 9.2 & \\
\hline & m 1iAb 1trat111AP1 & 8 & \\
\hline & mliAb1trat111AP1 & 9.7 & \\
\hline & mliAb1trat111AP1 & 10.7 & \\
\hline & m liAb1trat111AP1 & 7.6 & \\
\hline & miAb1trat111AP1 & 7.5 & \\
\hline & m1iAb1trat111AP1 & 9.4 & \\
\hline & m1iAb1trat111AP1 & 8.3 & \\
\hline & m 1iAb1trat111AP1 & 6.3 & \\
\hline & m1iAb1trat111AP1 & 10.1 & \\
\hline & m1iAb1trat111AP1 & 8.5 & \\
\hline & m1iAb1trat111AP1 & 9.5 & \\
\hline & m liab1trat111AP1 & 8.7 & \\
\hline & m1iAb1trat111AP1 & 6.6 & \\
\hline & m 1iAb1trat111AP1 & 9.6 & \\
\hline & m1iAb1trat111AP1 & 7.5 & \\
\hline & m1iAb1trat111AP1 & 8.4 & \\
\hline & m 1iAb1trat111AP1 & 8.1 & - \\
\hline
\end{tabular}

Figura 2. Tela de manipulação dos dados no FitFD.

Figure 2. Screen of data manipulation in FitFD.

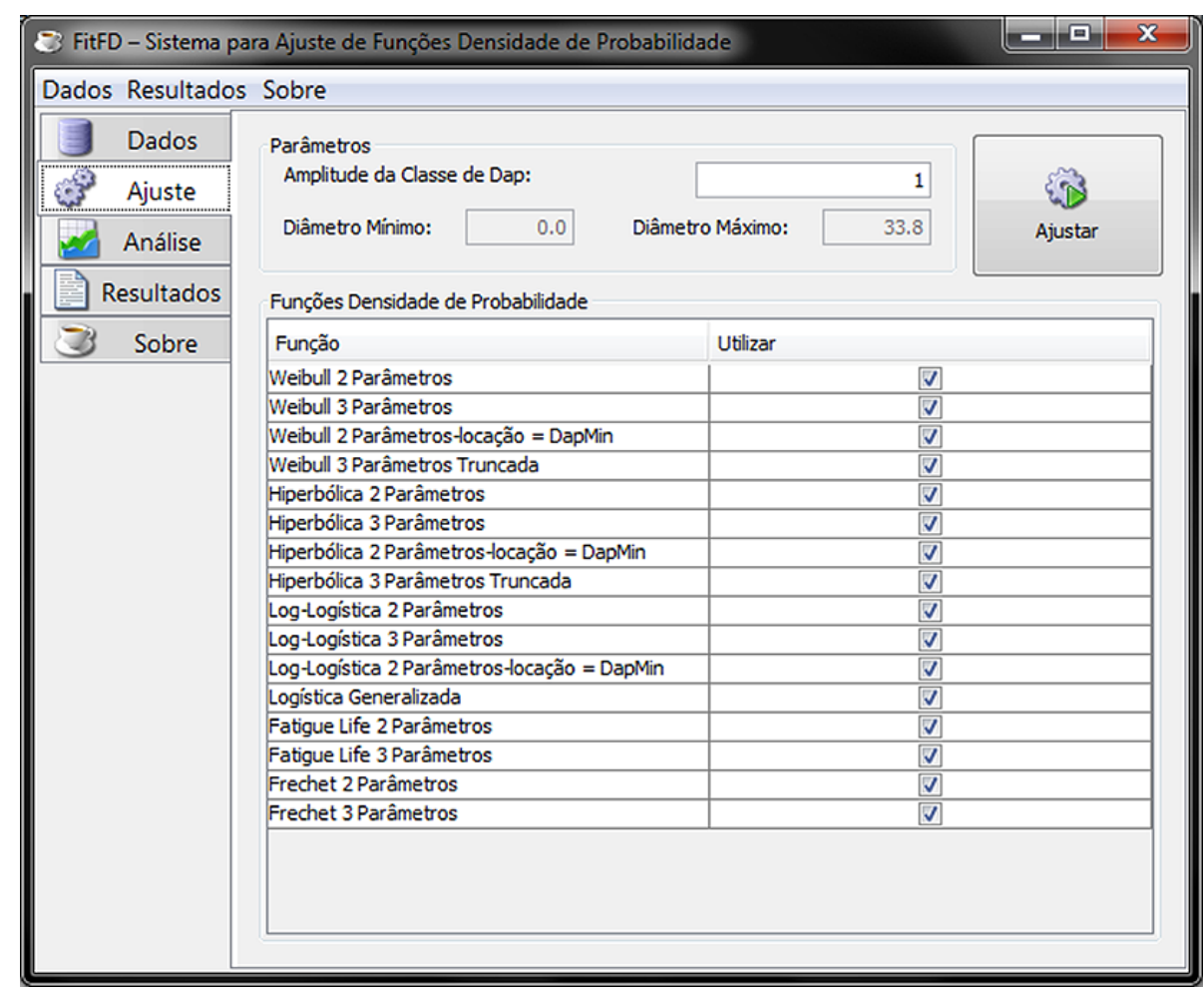

Figura 3. Tela de definição dos modelos de taper.

Figure 3. Screen definition of models of taper. 


\begin{tabular}{|c|c|c|c|c|c|c|c|c|c|}
\hline \multicolumn{7}{|c|}{8 FitFD - Sistema para Ajuste de Funções Densidade de Probabilidade } & \multicolumn{3}{|c|}{\begin{tabular}{|l|l|}
0 & 回 \\
\end{tabular}} \\
\hline \multicolumn{10}{|c|}{ Dados Resultados Sobre } \\
\hline Dados & \multicolumn{9}{|c|}{ Chave Dados } \\
\hline 69 Daus & \multicolumn{3}{|c|}{ m1iAb2trat124AP4 } & & & & & & \\
\hline E & & & & & & & & & \\
\hline An Análise & \multicolumn{9}{|l|}{ Dados } \\
\hline \multirow{2}{*}{ Resultados } & Dados & Gráfico & & & & & & & \\
\hline & \multicolumn{2}{|c|}{ Classe de ... } & Frequência... & Weibull 2 P... & Weibull $3 \mathrm{P} \ldots$ & Weibull 2 P... & Weibull 3 P... & Hiperb & \multirow{2}{*}{ 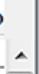 } \\
\hline \multirow{21}{*}{ (3) Sobre } & & 0.5 & 0 & 0 & 0 & 0 & 0 & & \\
\hline & & 1.5 & 0 & 0 & 0 & 0 & 0 & & \multirow{10}{*}{$\equiv$} \\
\hline & & 2.5 & 0 & 0 & 0 & 0 & 0 & & \\
\hline & & 3.5 & 0 & 0 & 0 & 0 & 0 & & \\
\hline & & 4.5 & 0 & 0,001 & 0,001 & 0 & 0 & & \\
\hline & & 5.5 & 0 & 0,003 & 0,003 & 0 & 0,002 & & \\
\hline & & 6.5 & 0,04 & 0,012 & 0,012 & 0,003 & 0,011 & & \\
\hline & & 7.5 & 0,049 & 0,037 & 0,037 & 0,025 & 0,037 & & \\
\hline & & 8.5 & 0,123 & 0,099 & 0,099 & 0,094 & 0,1 & & \\
\hline & & 9.5 & 0,173 & 0,209 & 0,209 & 0,219 & 0,213 & & \\
\hline & & 10.5 & 0,306 & 0,312 & 0,312 & 0,318 & 0,315 & & \\
\hline & & 11.5 & 0,269 & 0,255 & 0,255 & 0,25 & 0,255 & & \\
\hline & & 12.5 & 0,04 & 0,07 & 0,07 & 0,082 & 0,072 & & \\
\hline & & 13.5 & 0 & 0,003 & 0,003 & 0,008 & 0,003 & & \\
\hline & & 14.5 & 0 & 0 & 0 & 0 & 0 & & \\
\hline & & 15.5 & 0 & 0 & 0 & 0 & 0 & & \\
\hline & & 16.5 & 0 & 0 & 0 & 0 & 0 & & \\
\hline & & 17.5 & 0 & 0 & 0 & 0 & 0 & & \\
\hline & & 18.5 & 0 & 0 & 0 & 0 & 0 & & \\
\hline & & 19.5 & 0 & 0 & 0 & 0 & 0 & & + \\
\hline & 1 & & & III & & & & 1 & \\
\hline
\end{tabular}

Figura 4. Tela de análise entre valores observados e estimados.

Figure 4. Screen analysis between observed and estimated values.

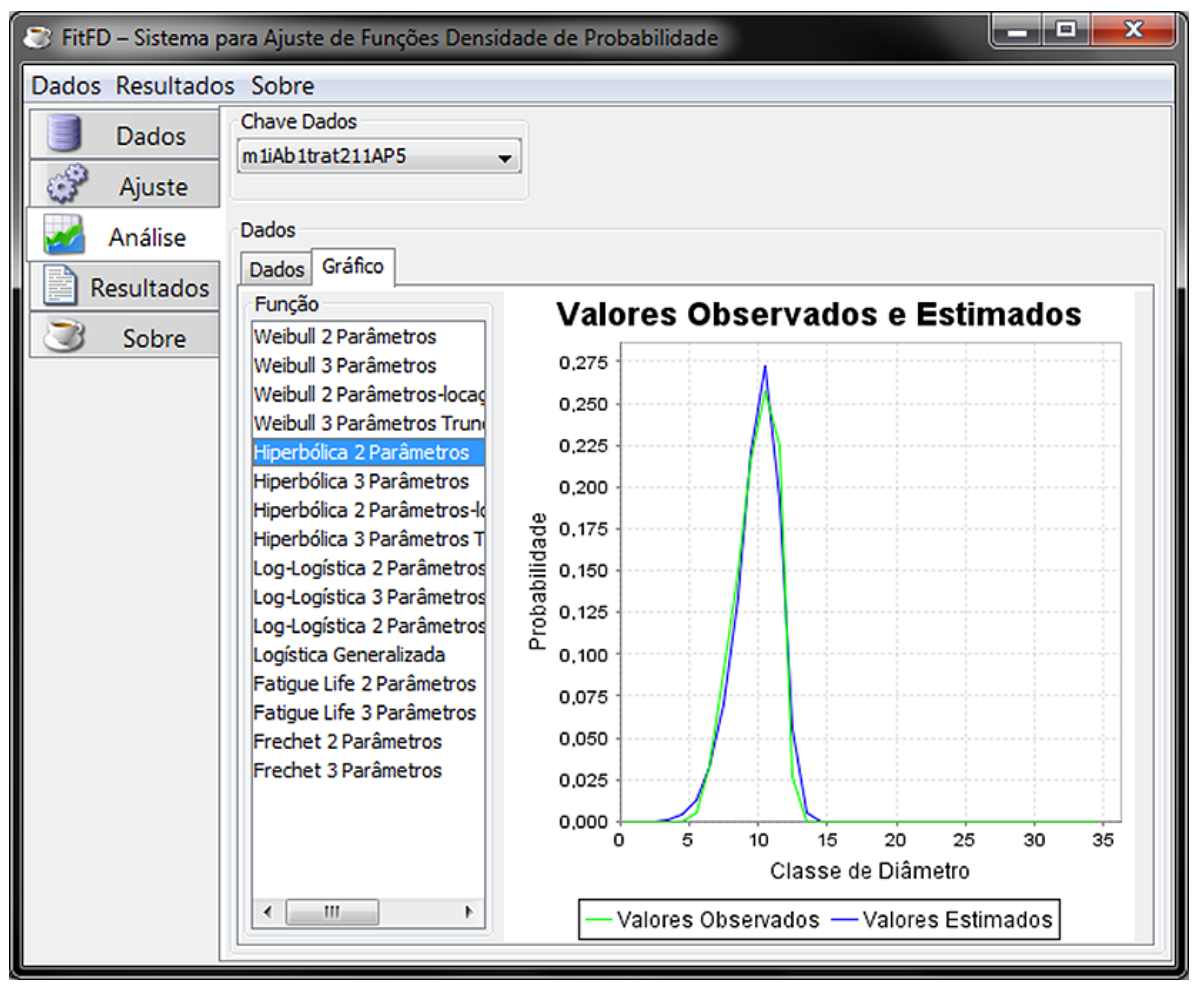

Figura 5. Tela de análise gráfica entre valores observados e estimados.

Figure 5. Screen analysis graphical between observed and estimated values. 


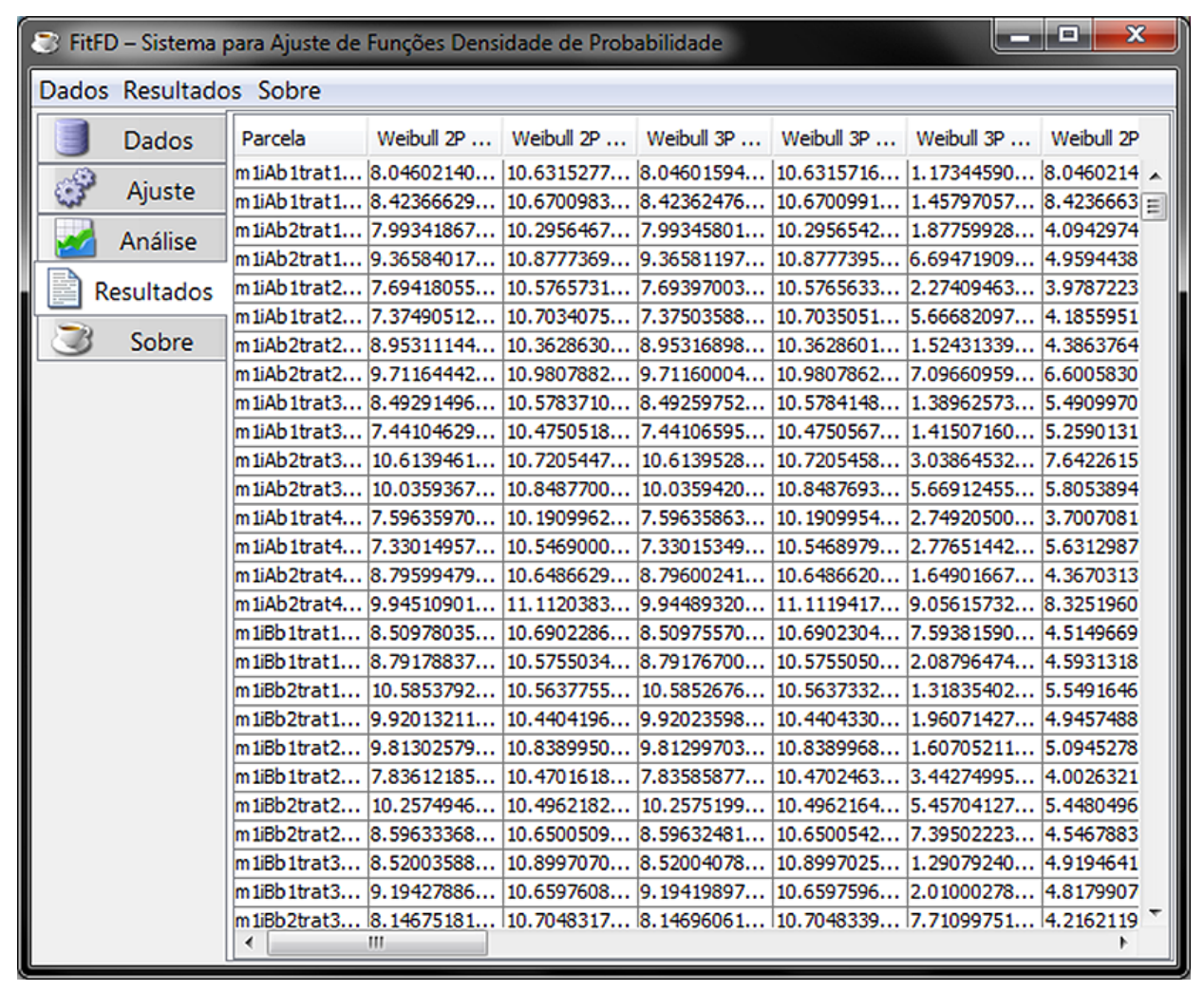

Figura 6. Resultados obtidos para cada parcela e função cadastrada.

Figure 6. Results obtained for each plot and registered function.

Os parâmetros estimados, bem como as estimativas por classe de diâmetro, podem ser exportados no menu Resultados.

O sistema desenvolvido neste trabalho auxilia os usuários na definição e escolha das $f d p$ que melhor atendam suas necessidades, contudo melhorias são necessárias. Como propostas para o desenvolvimento de novos recursos para o FitFD destacam-se:

- Permitir uma maior eficiência na manipulação dos dados observados, facilitando estratificações e junções de estratos dentro do sistema;

- Integração do sistema com ferramentas de cadastro e inventário florestal, tornando o sistema mais versátil e dinâmico;

- Geração de relatórios em formato padronizado de uma maneira fácil para o usuário;

- Integração com SGBD (Sistemas de Gerenciamento de Bancos de Dados), como o MySQL, Firebird, PostgreSQL, dentre outros, permitindo a exportação e publicação de informações em bancos de dados corporativos;

- Melhorar a ferramenta de análise gráfica, permitindo a visualização de várias funções em um mesmo gráfico.

\section{CONCLUSÃO}

O projeto iniciado mostrou-se eficiente para ajustes de funções de densidade probabilidade. O sistema desenvolvido está disponível na página do projeto NeuroForest.

\section{STATUS DA SUBMISSÃO}

Recebido: 11 mar., 2014

Aceito: 25 mar., 2016

\section{AUTOR(ES) PARA CORRESPONDÊNCIA}

\section{Mayra Luiza Marques da Silva}

Departamento de Ciências Florestais e da Madeira, Universidade Federal do Espírito Santo - UFES, Av. Governador Lindemberg, 316, Centro, CEP 29550-000, Jerônimo Monteiro, ES, Brasil

e-mail: mayralmsilva@gmail.com 


\section{REFERÊNCIAS}

Achar JA. Inference for Birnbaum-Saunders fatigue life model using Bayesian method. Computational Statistics \& Data Analysis 1993; 15(4): 367-380. http://dx.doi. org/10.1016/0167-9473(93)90170-X.

Binoti DHB, Binoti MLMS, Leite HG, Fardin LP, Oliveira JC. Probability density functions for description of diameter distribution in thinned stands of Tectona grandis. Cerne 2012; 18(2): 185-196. http://dx.doi.org/10.1590/S010477602012000200002 .

Binoti DHB. Funções densidade de probabilidade para a descrição da distribuição diamétrica de povoamentos desbastados de Tectona grandis [monografia]. Viçosa: Departamento de Engenharia Florestal, Universidade Federal de Viçosa; 2008.

Birnbaum ZW, Saunders SC. A new family of life distributions. Journal of Applied Probability 1969a; 6(02): 319-327. http://dx.doi.org/10.1017/S0021900200032848.

Birnbaum ZW, Saunders SC. Estimation for a family of life distributions with applications to fatigue. Journal of Applied Probability 1969b; 6(02): 328-347. http://dx.doi. org/10.1017/S002190020003285X.

Campos JCC, Leite HG. Mensuração florestal: perguntas e respostas. 3. ed. Viçosa: Editora UFV; 2009. 548 p.
Clutter JL, Fortson JC, Pienaar LV, Brister GH, Bailey RL. Timber management: a quantitative approach. New York: Wiley; 1983.

Horstmann CS, Cornell G. Core Java. 8. ed. São Paulo: Pearson Pretince Hall, 2010. 383 p. vol. 1 - Fundamentos.

Leite HG, Binoti DHB, Guimarães DP, Silva MLM, Garcia SLR. Avaliação do ajuste das funções Weibull e hiperbólica a dados de povoamentos de eucalipto submetidos a desbaste. Revista Árvore 2010; 34(2): 305-311. http:// dx.doi.org/10.1590/S0100-67622010000200013.

Nadarajah S, Kotz S. A Generalized logistic distribution. International Journal of Mathematics and Mathematical Sciences 2005; 19(19): 3169-3174. http://dx.doi.org/10.1155/ IJMMS.2005.3169.

Ng HKT, Kundu D, Balakrishnan N. Modified moment estimation for the two-parameter Birnbaum-Saunders distribution. Computational Statistics \& Data Analysis 2003; 43(3): 283-298. http://dx.doi.org/10.1016/S01679473(02)00254-2.

Wang ZH, Desmond AF, Lu XW. Modified censored moment estimation for the two-parameter BirnbaumSaunders distribution. Computational Statistics \& Data Analysis 2006; 4(4): 1033-1051. http://dx.doi.org/10.1016/j. csda.2004.10.014. 